Departamentos de Dermatología y

${ }^{2}$ Anatomía Patológica,

Facultad de Medicina,

Pontificia Universidad

Católica de Chile. Santiago de Chile.

alnterna de Medicina de la Universidad de Los Andes. Santiago de Chile.

Recibido el 8 de abril de 2011, aceptado el 2 de agosto de 2011

Los autores declaran no tener conflicto de intereses en este manuscrito.

Correspondencia a: Dra. Montserrat Molgó N. Dirección Dermatología,

Centro Médico San Joaquín. Av. Vicuña Mackenna 4686, Macul, Santiago, Chile.

Teléfono: (56 2) 3548659 , celular: 098258939

Fax: (56 2) 5529974.

Correo electrónico:

montsemolgo1@gmail.

com

\section{Síndrome de Sweet asociado a leucemia mieloide aguda y factor estimulante de colonias de granulocitos. Caso clínico}

\author{
MONTSERRAT MOLGÓ N. ${ }^{1}$, PILAR DE LA SOTTA F. ${ }^{1}$, \\ LAURA GIESEN F. ${ }^{\text {a }, ~ S E R G I O ~ G O N Z A ́ L E Z ~ B . ~}{ }^{2}$
}

\section{Sweet's syndrome associated with acute myeloid leukemia. Report of one case}

Sweet's syndrome, also known as acute febrile neutrophilic dermatosis, is characterized by fever, neutrophilia, erythematous and tender skin lesions that typically show a diffuse infiltrate of neutrophils in the upper dermis. This disorder has been associated with myeloproliferative syndromes. We report the case of a 53-year-old woman with an acute myeloid leukemia, presenting a Sweet's syndrome. A worsening of cutaneous lesions injuries was observed when granulocyte colony stimulating factor was added to treatment.

(Rev Med Chile 2011; 139: 1592-1596).

Key words: Granulocyte macrophage colony-stimulating factors, recombinant; Leukemia, myeloid, acute; Sweet syndrome.
$\mathrm{E}$ 1 síndrome de Sweet fue descrito originalmente por Robert Douglas Sweet en 1964 con el nombre de dermatosis neutrofílica aguda febril ${ }^{1,2}$. Es una enfermedad inflamatoria poco frecuente, con una incidencia cercana de 2,7 a $3 / 10^{6}$ casos anuales en la población general ${ }^{24}$. Se caracteriza por el comienzo brusco de fiebre, leucocitosis y lesiones cutáneas, que son típicamente caracterizadas como pápulas, placas o nódulos eritematosos, bien delimitados, sensibles al tacto, con o sin pseudovesículas, que muestran un sustrato microscópico de denso infiltrado neutrofílico de la dermis papilar; sin vasculitis ${ }^{2}$. Las lesiones pueden aparecer en cualquier parte del cuerpo, pero predominan en la parte alta incluyendo la cara. $\mathrm{La}$ fisiopatología del síndrome de Sweet sigue siendo desconocida, pero se sabe que las citoquinas juegan un rol importante en su etiopatogenia ${ }^{2}$.

Se desconoce la etiología de este síndrome. Se han descrito casos asociados a neoplasias hematológicas, tumores sólidos, enfermedades inmunológicas, enfermedades intestinales y fármacos, siendo el factor estimulante de colonias de granulocitos (G-CSF), el fármaco más frecuentemente reportado ${ }^{3-5}$.

Los corticoides sistémicos son en la actualidad el gold standard del tratamiento ${ }^{1}$.

Se reporta el caso de una paciente con síndrome de Sweet y leucemia mieloide aguda con lesiones cutáneas que se agravaron cuando se administró G-CSF.

\section{Caso clínico}

Paciente de sexo femenino de 53 años de edad, previamente sana, con historia de tres semanas de evolución de compromiso del estado general, fatiga, pérdida de peso, cefalea y mialgia generalizada. A la semana siguiente, la paciente evolucionó con placas y pápulas eritematosas, dolorosas, que predominaban en cara, cuello, brazos y piernas.

De los exámenes de laboratorio, el hemograma mostraba neutropenia, trombocitopenia, anemia 
y blastos. Se confirmó el diagnóstico de leucemia mieloide aguda por medio del estudio de la médula ósea, por lo que se decidió iniciar quimioterapia de inducción con daunorubicina y citarabina y terapia adyuvante con Filgrastim ${ }^{\circledR}$ (G-CSF) para el manejo de la neutropenia febril asociada.

A los 13 días post-quimioterapia, junto con un aumento del número de neutrófilos, la paciente presentó un incremento de lesiones, en forma de ampollas y bulas hemorrágicas rodeadas por placas inflamatorias muy sensibles localizadas en cara, cuello y extremidades, que se asociaban a fiebre alta, artralgias y mialgias (Figura 1). Se descartó por medio de exámenes y cultivos del líquido de las pseudoampollas, la presencia de infecciones virales, micóticas y bacterianas.

La paciente persistió con fiebre, cefalea y evolucionó con conjuntivitis y erosiones en mucosa oral y lengua, asociadas a hiperplasia gingival (Figura 2). Se realizó una biopsia de las lesiones cutáneas, que mostró edema y un denso infiltrado neutrofílico en la dermis, sin signos de vasculitis, compatible con síndrome de Sweet (Figura 3). Producto de lo anterior, se comenzó tratamiento con hidrocortisona intravenosa, que se asoció a una mejoría significativa de las lesiones cutáneas (Figura 4). Sin embargo, la paciente falleció antes de comenzar el segundo ciclo de la quimioterapia, debido a una insuficiencia cardiaca y respiratoria. No se obtuvo autopsia.

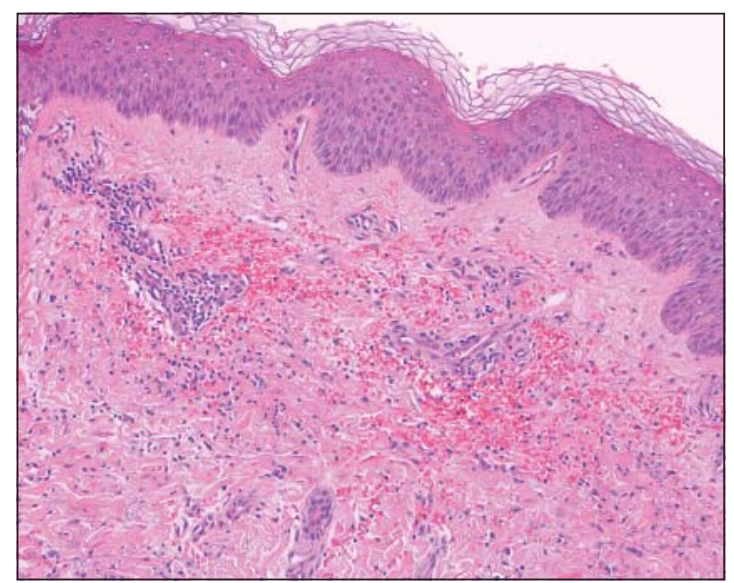

Figura 3. Infiltrado neutrofílico dérmico difuso, con hemorragia; HE, 100x (HE: Hematoxilina-Eosina (tinción), 100x: Ampliación 100x).

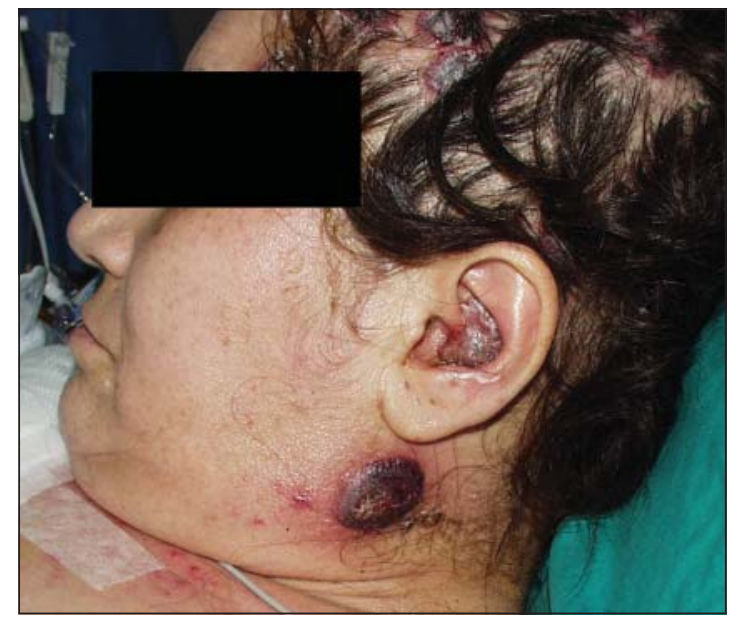

Figura 1. Ampollas y bulas hemorrágicas.

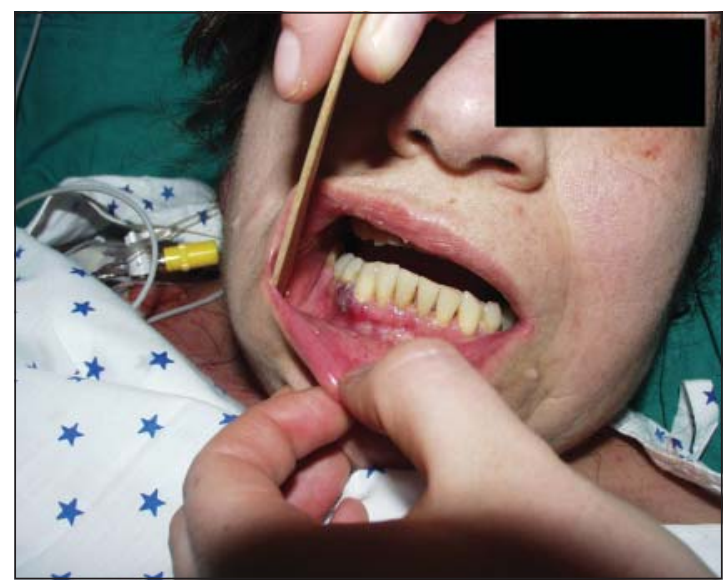

Figura 2. Erosiones de la mucosa oral.

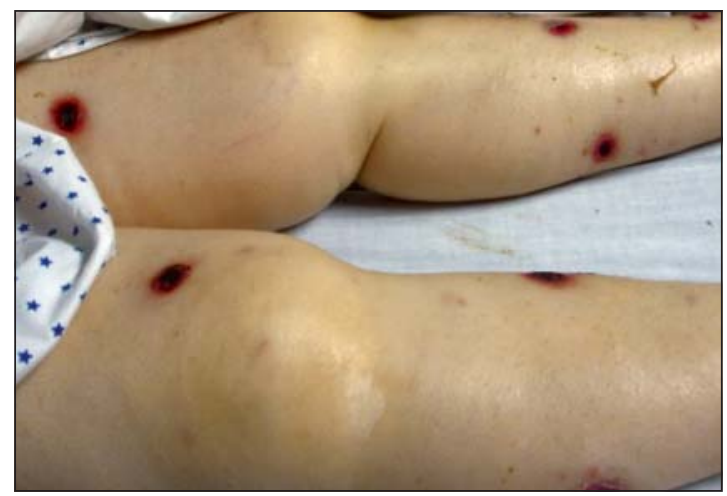

Figura 4.Lesiones cutáneas en regresión. 


\section{Discusión}

El síndrome de Sweet se caracteriza por una constelación de síntomas clínicos. La fiebre es el síntoma más comúnmente descrito ${ }^{1,6}$. Las lesiones cutáneas primarias son pápulas o nódulos eritematosos muy sensibles, no pruriginosos, de 2 a $10 \mathrm{~cm}$ de diámetro, que normalmente aparecen como lesiones múltiples que se distribuyen de forma asimétrica en cara, cuello, brazos y tronco superior, pudiendo coalecer y formar placas irregulares, de bordes netos, con pseudovesículas, pseudopústulas y/o pústulas en la superficie $e^{3,6,7}$. Otros síntomas asociados son: malestar general, artralgia, mialgia, conjuntivitis, y ocasionalmente compromiso renal ${ }^{1,4,5,8-10}$. Varios de estos síntomas estuvieron presentes en nuestra paciente.

La fisiopatología del síndrome de Sweet sigue siendo desconocida, postulándose una asociación con depósito de complejos inmunes, activación de linfocitos T, función neutrofílica alterada y, sobre todo, una desregulación en la secreción de G-CSF, del factor estimulante de colonias de granulocitos y macrófagos (GM-CSF), interferón gamma y citoquinas, las cuales estarían estimuladas por un mecanismo de hipersensibilidad, precipitando la activación de neutrófilos y su infiltración en la dermis papilar ${ }^{2,19,25}$.

El síndrome de Sweet se puede clasificar, dependiendo de las patologías asociadas, en tres condiciones clínicas: síndrome de Sweet clásico o idiopático, síndrome de Sweet asociado a malignidad y el síndrome de Sweet inducido por fármacos ${ }^{1,2,13}$.

El síndrome de Sweet clásico, presente en 71\% del total de casos, es más frecuente en mujeres (3:1) y su edad media de presentación es entre los 30 y 50 años. Se puede asociar a infecciones respiratorias altas, enfermedades autoinmunes, enfermedades inflamatorias intestinales y embarazo ${ }^{1,3,4,5,13}$. Su forma de presentación es característica y se asocia a una rápida remisión después del tratamiento con corticoides sistémicos, presentando cerca de 30\% de los pacientes una o múltiples recidivas.

Entre 15 y 20\% de los casos se presenta como síndrome de Sweet asociado a malignidad, donde la dermatosis puede aparecer antes, durante o después del diagnóstico del cáncer. La incidencia por sexo suele ser similar ${ }^{14,15}$. El 85\% corresponde a neoplasias hematológicas, siendo las principales en orden de frecuencia: la leucemia mieloide aguda
(42\%), como era el caso de nuestra paciente, los linfomas (11\%), los síndromes mielodisplásicos (9\%) y la leucemia mielocítica crónica (7\% de los casos). El 15\% restante, lo constituyen los tumores sólidos asociados al síndrome de Sweet, siendo los más frecuentes los carcinomas del tracto genitourinario, mama y tracto gastrointestinal ${ }^{15}$.

En pacientes con trastornos hematológicos, el síndrome de Sweet se puede manifestar de diferentes formas: ya sea, como un síndrome paraneoplásico, una dermatosis inducida por fármacos o como una condición en que las lesiones de la piel ocurren simultáneamente con una leucemia. Las ampollas, las bulas hemorrágicas y las lesiones ulcerosas de la mucosa oral, ocurren con más frecuencia en estos pacientes y la neutropenia y la anemia no son inusuales. Por otra parte, un tercio de los pacientes presentan episodios recurrentes ${ }^{16}$.

A pesar de que la leucocitosis con predominio de neutrófilos es considerada como un criterio diagnóstico para el síndrome de Sweet, ésta puede no estar presente, lo cual no excluye su diagnóstico ${ }^{17}$. Pacientes con enfermedades hemato-oncológicas frecuentemente tienen anemia, leucopenia y plaquetopenia, dadas por la evolución natural de la enfermedad o por el tratamiento con quimioterapia. Es así, como en nuestra paciente se pesquisó una neutropenia durante el diagnóstico del síndrome de Sweet, corroborando que este síndrome debe sospecharse como un diagnóstico diferencial, en todo paciente que presente lesiones cutáneas dolorosas, independiente del recuento de células sanguíneas.

El síndrome de Sweet inducido por fármacos, se ha asociado principalmente al tratamiento con G-CSF, como en el caso de nuestra paciente. El G-CSF es usado comúnmente como tratamiento de la mielosupresión desencadenada en pacientes que están bajo quimioterapia de inducción, describiéndose diversos efectos adversos secundarios a su uso, como la vasculitis leucocitoclástica y el síndrome de Sweet, entre otros ${ }^{4,5,18}$. Una vez suspendida la administración del fármaco, se evidencia una rápida mejoría del cuadro. Pese a que se conoce la asociación del G-CSF con el síndrome de Sweet, aún no es ampliamente reconocida, no siendo considerada como diagnóstico diferencial frente a presentaciones clínicas inusuales.

En la actualidad existe una serie de criterios diagnósticos para el síndrome de Sweet, siendo necesaria la presencia de dos criterios mayores $y$, 
Tabla 1. Criterios diagnósticos para el síndrome de Sweet

Mayores

1) Aparición súbita de placas o nódulos eritematosos o violáceos y dolorosos

2) Infiltración dérmica de PMN neutrófilos sin vasculitis leucocitoclástica

Menores

1) Pródromos de fiebre o de proceso infeccioso

2) Leucocitosis

3) Asociación de artralgias, conjuntivitis, fiebre o neoplasia subyacente

4) Respuesta a los corticosteroides sistémicos

5) Aumento de la velocidad de eritrosedimentación

Tabla 2. Criterios diagnósticos para el síndrome de Sweet inducido por drogas

Clínicos:
Placas o nódulos eritematosos y dolorosos de aparición
súbita
Histopatológicos:
Infiltrado neutrofílico denso sin evidencia de vasculitis
Constitucionales:
Fiebre $>38^{\circ} \mathrm{C}$
Otros:
1. Relación temporal entre la toma del fármaco y la apa-
rición de las lesiones o recurrencia de síntomas con la
reexposicón al fármaco
2. Resolución tras la suspensión del fármaco o después
del tratamiento con corticosteroides, sin recidivas.

por lo menos, dos menores, para hacer el diagnóstico definitivo (Tabla 1$)^{15,23,27}$.

En el caso del síndrome de Sweet inducido por fármacos, existen algunas diferencias respecto a los criterios diagnósticos menores del síndrome de Sweet clásico, como la relación temporal entre la toma del fármaco y la aparición de las lesiones, y su resolución en forma espontánea o con tratamiento con corticoides, tras la suspensión del fármaco; sin presencia de recidivas (Tabla 2$)^{26}$.

El diagnóstico histológico se caracteriza por un denso infiltrado de neutrófilos maduros en la dermis papilar, asociado a edema, con ausencia de granulomas y sin signos de vasculitis leucocitoclástica ${ }^{11,12}$.

Los corticoides sistémicos son en la actualidad el tratamiento de elección para los síntomas cu- táneos y sistémicos ${ }^{1}$, respondiendo rápidamente la gran mayoría de los pacientes, a pesar de que algunos casos tienden a recidivar en el tiempo. Para aquellos casos recurrentes o refractarios al uso de corticoides, se ha planteado el uso de antiinflamatorios no esteroidales, colchicina, dapsona, doxiciclina, entre otros, y más recientemente, el uso de etanercept, un antagonista del factor de necrosis tumoral alpha (TNF- $\alpha$ ), que inhibiría la activación característica de los neutrófilos en el síndrome de Sweet. A su vez, estudios recientes han revelado que el uso de inmunoglobulinas endovenosas, IVIG, en asociación con corticoides y otros agentes antiinflamatorios, podrían ser efectivas en aminorar el curso clínico de la enfermedad ${ }^{20-22}$.

Es necesario acentuar la importancia de considerar el síndrome de Sweet en el diagnóstico diferencial de pacientes con leucemia mieloide aguda, con lesiones cutáneas atípicas, difíciles de diferenciar de otras condiciones y en aquellos pacientes tratados con G-CSF, siendo fundamentales para el diagnóstico definitivo los hallazgos histopatológicos de la biopsia junto con la clínica del paciente.

\section{Referencias}

1. Cohen PR. Sweet's syndrome: a comprehensive review of an acute febrile neutrophilic dermatosis. Orphanet J Rare Dis 2007; 2: 34.

2. Mahajan VK., Sharma NL, Sharma RC. Sweet's syndrome from an Indian perspective: a report of four cases and review of the literature. Int J of Dermatol 2006; 45 (6): 702-8.

3. Al-Saad K, Khanani MF, Naqvi A, Krafchik B, Grant R, Pappo A. Sweet Syndrome developing during treatment with all-trans retinoic acid in a child with acute myelogenous leukemia. J Pediatr Hematol Oncol 2004; 26 (3): 197-9.

4. Thompson MA, Dyson SW, Faderl S. Sweet's syndrome in chronic lymphocytic leukemia associated with neutropenic fever and granulocyte colony stimulation factor. Am J Hematol 2006; 81 (9): 703-5.

5. Kumar G, Bernstein JM, Waibel JS, Baumann MA. Sweet's syndrome associated with sargramostim (granulocyte-macrophage colony stimulating factor) treatment. Am J Hematol 2004; 76(3): 283-5.

6. Cohen PR, Kurzrock R. Sweet's syndrome revisited: a review of disease concepts. Int J Dermatol 2003; 42 (10): 761-78. 
7. Cohen PR, Almeida L, Kurzrock R. Acute febrile neutrophilic dermatosis. Am Fam Physician 1989; 39( 3): 199-204.

8. Cohen PR, Kurzrock R. Sweet's syndrome: a neutrophilic dermatosis classically associated with acute onset and fever. Clin Dermatol 2000; 18 (3): 265-82.

9. Wallach D. Neutrophilic dermatoses: an overview. Clin Dermatol 2000; 18(3): 229-31.

10. Wallach D, Vignon-Pennamen MD. From acute febrile neutrophilic dermatosis to neutrophilic disease: forty years of clinical research. J Am Acad Dermatol 2006; 55 (6): 1066-71.

11. Margo CM, De Moraes E, Burns F. Sweet's syndrome in the setting of CD34-positive acute myelogenous leukemia treated with granulocyte colony stimulating factor: evidence for a clonal neutrophilic dermatosis. J Cutan Pathol 2001; 28 (2): 90-6.

12. Malone JC, Stone SP. Sweet syndrome: a disease in histologic evolution? Arch Dermatol 2005 Jul, 141(7): 893-5.

13. Cohen PR, Kurzrock R. Sweet's syndrome and cancer. Clin Dermatol 1993 Jan-Mar; 11(1): 149-57. Disponible en: www.pubmed.com (consultado el 10 de marzo de 2011).

14. Haverstock C, Libecco JF, Sadeghi P, Maytin E. Tender erythematous plaques in a woman with acute myelogenous leukemia. Arch Dermatol 2006; 142 (2): 235-40.

15. Cohen PR. Neutrophilic dermatoses occurring in oncology patients. Int J Dermatol 2007; 46 (1): 106-11.

16. Walker DC, Cohen PR. Trimethoprim-sulfamethoxazole-associated acute febrile neutrophilic dermatosis: case report and review of drug-induced Sweet's syndrome. J Am Acad Dermatol 1996; 34: 918-23.

17. Disel U, Paydas S, Yavuz S, Tuncer I, Alpay R. Bilateral ear Sweet's syndrome in a case with relapse acute mye- loblastic leukemia. Leuk Res 2006; 30 (3): 364.

18. Arbetter KR, Hubbard KW, Markovic SN, Gibson LE, Phyliky RL. Case of granulocytic colony-stimulating factor-induced Sweet's syndrome. Am J Hematol 1999; 61 (2): 126-9.

19. Reuss-Borst MA, Pawelec G, Saal JG, Horny HP, Muller CA, Waller HD. Sweet's syndrome associated with myelodysplasia: possible role of cytokines in the pathogenesis of the disease. Br J Haematol 1993; 84 (2): 356-8.

20. Guhl G, García-Diez A. Subcutanous sweet syndrome. Dermatol Clin 2008; 26 (4): 541-51.

21. Yamauchi PS, Turner L, Lowe NJ, Gindi V, Jackson JM. Treatment of recurrent Sweet's syndrome with coexisting rheumatoid arthritis with the tumor necrosis factor antagonist etanercept. J Am Acad Dermatol 2006; 54 (3 Suppl 2): S 122-6.

22. Gill HH, Leung AY, Trendell-Smith NJ, Yeung CK, Liang R. Sweet Syndrome due to Myelodysplastic Syndrome: Possible Therapeutic Role of Intravenous Immunoglobulin in Addition to Standard Treatment. Adv Hematol 2010; 2010: 328316: 1-3.

23. Ribeiro A, Costa J, Bogas M, Costa L, Araújo D. Acute febrile neutrophilic dermatosis-Sweet's syndrome. Acta Reumatol Port 2009; 34 (3): 536-40.

24. Djien V, Wallach D. Sweet's syndrome: practical conduct. Ann Dermatol Venereol 1999; 126 (4): 343-7.

25. Burrall B. Sweet's syndrome (acute febrile neutrophilic dermatosis). Dermatol Online J 1999; 5 (1): 8.

26. Del Pozo J, Martínez W, Fonseca E. Síndrome de Sweet y enfermedad sistémica. Piel 2004; 19: 135-47.

27. Davoli D, Chavero I, Kantor B, García H, Redin I, Celentano A, Cera D. Síndrome de Sweet em Tricoleucemia asociado a Factores Estimulantes de Colonias. Rev Med Rosario 2008; 74: 26-31. 